\title{
ZERO CYCLES ON QUADRIC HYPERSURFACES
}

\author{
RICHARD G. SWAN \\ (Communicated by Louis J. Ratliff, Jr.)
}

\begin{abstract}
Let $X$ be a projective quadric hypersurface over a field of characteristic not 2. It is shown that the Chow group $A_{0}(X)$ of 0 -cycles modulo rational equivalence is infinite cyclic, generated by any point of minimal degree.
\end{abstract}

Let $k$ be a field of characteristic not 2 and let $X \subset \mathbb{P}_{k}^{d+1}$ be a quadric hypersurface defined by an equation $q=0$ where $q$ is a quadratic form in $d+2$ variables over $k$. In [4] I computed the $K$-theory of $X$ assuming that $q$ is nondegenerate. However the problem of computing the Chow groups of $X$, which was proposed in [3] is, to the best of my knowledge, still open. I will treat here the first nontrivial case by determining the Chow group $A_{0}(X)$ of 0 -cycles modulo rational equivalence [1]. The result turns out to hold also in the singular case. My original proof made use of the results of [4]. I would like to thank Mohan Kumar for pointing out that this was not necessary and that only elementary facts about $A_{0}$ are needed.

Theorem. Let $X \subset \mathbb{P}_{k}^{d+1}$ be defined by $q=0$ where $q$ is a quadratic form over a field $k$ of characteristic not 2 and $d>0$. Then $A_{0}(X)=\mathbb{Z}$. It is generated by any rational point if one exists. If $X$ has no rational point then $A_{0}(X)$ is generated by any point of degree 2 over $k$.

We can obviously assume that $q$ is not identically 0 so that $\operatorname{dim} X=d$. If $d=0$, it is then clear that $A_{0}(X)=\mathbb{Z} \times \mathbb{Z}$ if $X$ consists of two rational points and $A_{0}(X)=\mathbb{Z}$ otherwise. For the reader's convenience I will restate the following standard classification for the case $d=1$.

Lemma 1. Let $X \subset \mathbb{P}^{2}$ be a quadric curve. Then one of the following holds:

(1) $X=\mathbb{P}^{1}$ embedded in $\mathbb{P}^{2}$ by the Veronese embedding.

(2) $X$ is a smooth conic with no rational point.

(3) $X=L_{1} \cup L_{2}$ is a union of two lines defined over $k$ with one common point.

Received by the editors December 16, 1988 .

1980 Mathematics Subject Classification (1985 Revision). Primary 14C25; Secondary 11E04, $14 \mathrm{G} 05$. 
(4) $X=L \cup L^{\prime}$ where $L$ is a line defined over a quadratic extension of $k$ (but not over $k$ ) and $L^{\prime}$ is its conjugate. The only rational point on $X$ is the intersection $L \cap L^{\prime}$.

(5) $X$ is a double line defined by $l^{2}=0$ where $l$ is a linear form over $k$.

If $q$ is nondegenerate and nonisotropic we have case (2). In the isotropic case we can write $q=x y-z^{2}$, getting case (1). If $q$ reduces to $a x^{2}+b y^{2}$ with $a b \neq 0$, we have case (3) if $-a^{-1} b$ is a square in $k$, and case (4) otherwise. If $q$ reduces to $a x^{2}$ we have case (5).

I will write $x \sim y$ when $x$ and $y$ are rationally equivalent. In the following lemmas, $X$ will always denote the quadric hypersurface of the theorem.

Lemma 2. If $x$ and $y$ are rational points of $x$ then $x \sim y$.

Proof. Let $P$ be a 2-plane in $\mathbb{P}^{d+1}$ containing the points $x$ and $y$. Then $P \cong \mathbb{P}^{2}$. If $P \subset X$ the result is clear. Otherwise $X \cap P$ is as in Lemma 1 . In case (1) the result is again clear. Case (2) cannot occur. In case (3) $x$ and $y$ are rationally equivalent to the common point $a=L_{1} \cap L_{2}$. In case (4) there is only one rational point, so $x=y$. Finally, in case (5) $x$ and $y$ lie on the line $l=0$, so $x \sim y$.

Lemma 3. The theorem is true if $X$ has a rational point;

Proof. Let $x$ be a rational point and let $y$ be any point. Let $k^{\prime}=\kappa(y)$ be the residue field of $y$ and let $X^{\prime}=k^{\prime} \otimes_{k} X$ with projection $\pi: X^{\prime} \rightarrow X$. Then $X^{\prime}$ is just the quadric hypersurface defined by $q=0$ over $k^{\prime}$. Now $\pi^{-1}(y)=\operatorname{Spec} k^{\prime} \otimes_{k} \kappa(y)$ has a rational point $y^{\prime}$ and $\pi^{-1}(x)=\operatorname{Spec} k^{\prime} \otimes_{k} \kappa(x)=$ Spec $k^{\prime}=\left\{x^{\prime}\right\}$ with $x^{\prime}$ rational. By Lemma 2, $x^{\prime} \sim y^{\prime}$. Therefore $y=$ $\pi_{*}\left(y^{\prime}\right) \sim \pi_{*}\left(x^{\prime}\right)=\left|k^{\prime}: k\right| x$. Thus $A_{0}(X)$ is geneated by $x$. Since deg: $A_{0}(X) \rightarrow$ $\mathbb{Z}$ by $\operatorname{deg} z=|\kappa(z): k|$, the result follows.

We can now assume that $X$ has no rational point. Since we can write $q=\sum a_{i} x_{i}^{2}$, it is clear that $X$ has points of degree 2 . Also $X$ must be smooth since all $a_{i}$ must be nonzero. The following is a special case of [3, Lemma 13.4].

Lemma 4. If $X$ has no rational point the all points of $X$ have even degree.

Proof. Suppose $x \in X$ has odd degree. Let $k^{\prime}=\kappa(x)$. Then $k^{\prime} \otimes_{k} X$ has a rational point so that $q$ is isotropic over $k^{\prime}$. Since $\left|k^{\prime}: k\right|$ is odd, a theorem of Springer [2, Chap. 7, Theorem 2.3] implies that $q$ is isotropic over $k$, so $X$ has a rational point.

Lemma 5. Let $K$ be the kernel of $\operatorname{deg}: A_{0}(X) \rightarrow \mathbb{Z}$. Then $2 K=0$.

Proof. We can assume that $X$ has no rational point. Let $x \in X$ have degree 2 and set $k^{\prime}=\kappa(x)$. Let $X^{\prime}=k^{\prime} \otimes_{k} X$ with projection $\pi: X^{\prime} \rightarrow X$. Then $\pi^{-1}(:)=\operatorname{Spec} k^{\prime} \otimes_{k} \kappa(x)=\left\{x^{\prime}, x^{\prime \prime}\right\}$ where $x^{\prime}$ and $x^{\prime \prime}$ are rational over $k^{\prime}$. If $y$ is any closed point, $\pi^{-1}(y)=\operatorname{Spec} k^{\prime} \otimes_{k} \kappa(y)=$ either $\left\{y^{\prime}, y^{\prime \prime}\right\}$ or $\{z\}$ 
depending on whether $k^{\prime} \otimes_{k} \kappa(y)$ splits or not. By Lemma 3 we can write $y^{\prime} \sim m x^{\prime}$ or $z \sim m x^{\prime}$ getting either $y=\pi_{*}\left(y^{\prime}\right) \sim m \pi_{*}\left(x^{\prime}\right)=m x$ or $2 y=$ $\pi_{*}(z) \sim m x$. It follows that twice any 0 -cycle is rationally equivalent to a multiple of $x$.

Lemma 6. If $x$ and $y$ have degree 2 then $x \sim y$.

Proof. We can assume $X$ has no rational point by Lemma 3. Let $V=X(\bar{k})$ be the variety corresponding to $X$ over the algebraic closure $\bar{k}$ of $k$. The point $x$ corresponds to a pair of points $\xi, \xi^{\prime}$ of $V$. Since char $k \neq 2, \kappa(x)$ is Galois over $k$ so that $\xi$ and $\xi^{\prime}$ are distinct and conjugate over $k$. The line $L$ spanned by $\xi$ and $\xi^{\prime}$ is stable under the Galois group and therefore is defined over $k$. Since $(L \cdot X)=2$ by Bezout's theorem, we see that $L \cdot X=x$. Similarly, $y=L^{\prime} \cdot X$ for some line $L^{\prime}$ defined over $k$. Since $L \sim L^{\prime}$ as 1-cycles on $\mathbb{P}^{d+1}$, it follows that $x \sim y$.

If $y$ is a closed point of $X$ I will say that $y$ is "good" if $y \sim m x$ for some integer $m$ and some point $x$ of degree 1 or 2 . The theorem will follow from Lemmas 3 and 6 if we can show that all points are good.

Lemma 7. Let $\pi: X^{\prime}=k^{\prime} \otimes_{k} X \rightarrow X$ be the canonical projection where $\left|k^{\prime}: k\right|$ is odd. If all points of $\pi^{-1}(y)$ are good, so is $y$.

Proof. We can assume that $X$ has no rational points. The same is then true of $X^{\prime}$ by Springer's theorem as in the proof of Lemma 4. Therefore if $x$ is a point of degree 2 on $X$ then $\pi^{-1}(x)=\left\{x^{\prime}\right\}$, since otherwise $\pi^{-1}(x)$ would consist of two rational points.

Let $k^{\prime} \otimes_{k} \kappa(y)=A_{1} \times \cdots \times A_{r}$ where the $A_{i}$ are local artinian with residue fields $k_{i}=A_{i} / \mathfrak{M}_{i}=\kappa\left(y_{i}\right)$ where the $y_{i}$ are the points of $\pi^{-1}(y)$. Then $\left|k^{\prime}: k\right|=\operatorname{dim}_{\kappa(y)} k^{\prime} \otimes_{k} \kappa(y)=\sum l\left(A_{i}\right)\left|k_{i}: \kappa(y)\right|$ so for some $i,\left|\kappa\left(y_{i}\right): \kappa(y)\right|$ is odd. Since $y_{i}$ is good, $y_{i} \sim m x^{\prime}$ for some $m$ and hence $\pi_{*}\left(y_{i}\right)=$ $\left|\kappa\left(y_{i}\right): \kappa(y)\right| y \sim m \pi_{*}\left(x^{\prime}\right)=m|k /: k| x$. Since $\left|\kappa\left(y_{i}\right): \kappa(y)\right|$ is odd and $2 y \sim m^{\prime} x$ for some $m^{\prime}$ by Lemma 5 , the result follows.

Lemma 8. Let $\eta: X^{\prime}=k^{\prime} \otimes_{k} X \rightarrow X$ be the canonical projection where $\left|k^{\prime}: k\right|=$ 2. Let $y$ be a closed point of $X$ such that $\eta^{-1}(y)=\left\{y^{\prime}, y^{\prime \prime}\right\}$ has two distinct points. If $y^{\prime}$ is good, so is $y$.

Proof. We can assume that $X$ has no rational points. If $x^{\prime} \in X^{\prime}$ is rational, then $x=\eta_{*}\left(x^{\prime}\right)$ has degree 2 and $y^{\prime} \sim m x^{\prime}$ implies $y=\eta_{*}\left(y^{\prime}\right) \sim m x$. If $X^{\prime}$ has no rational point and $x \in X$ has degree 2, then $\eta^{-1}(x)=\left\{x^{\prime}\right\}$ where $x^{\prime}$ has degree 2 on $X^{\prime}$. Now $y^{\prime} \sim m x^{\prime}$, so $y=\eta_{*}\left(y^{\prime}\right) \sim m \eta_{*}\left(x^{\prime}\right)=2 m x$ as required.

We will now show that all closed points of $X$ are good. Let $y \in X$ have degree $n$. By induction, we can assume that all points of degree less than $n$ are good on all the hypersurfaces $k^{\prime} \otimes_{k} X$. Let $K$ be the "normal closure" of $\kappa(y)$, i.e., the composite of all conjugates of $\kappa(y)$ in the algebraic closure 
$\bar{k}$ of $k$. Let $G=\operatorname{Aut}(K / k)$. Then $K^{G}$ is purely inseparable over $k$ and therefore of odd degree since char $k \neq 2$. Let $H$ be a 2-Sylow subgroup of $G$. Then $k^{\prime}=K^{H}$ is of odd degree over $K^{G}$ and hence over $k$. Let $\pi: X^{\prime}=k^{\prime} \otimes_{k} X \rightarrow X$ be the canonical projection. By Lemma 7 it will suffice to show that each point $y^{\prime}$ of $\pi^{-1}(y)$ is good. Now $\kappa\left(y^{\prime}\right)$ is a quotient of $k^{\prime} \otimes_{k} \kappa(y)$. Any embedding of $\kappa\left(y^{\prime}\right)$ in $\bar{k}$ fixing $k^{\prime}$ will necessarily send $\kappa(y)$ into $K$ so we get $k^{\prime}=K^{H} \subset \kappa\left(y^{\prime}\right) \subset K$ and $\kappa\left(y^{\prime}\right)=K^{L}$ for some subgroup $L$ of $H$. If $L=H$ then $y^{\prime}$ is rational and hence good. If $L<H$, let $M$ be a maximal proper subgroup of $H$ containing $L$. Then $|H: M|=2$. Let $k^{\prime \prime}=K^{M}$ and consider $\eta: X^{\prime \prime}=k^{\prime \prime} \otimes_{k} X \rightarrow X^{\prime}$. Since $k^{\prime \prime}=K^{M} \subset K^{L}=\kappa\left(y^{\prime}\right)$ and $\left|k^{\prime \prime}: k^{\prime}\right|=2, k^{\prime \prime} \otimes_{k^{\prime}} \kappa\left(y^{\prime}\right) \approx \kappa\left(y^{\prime}\right) \times \kappa\left(y^{\prime}\right)$ so $\eta^{-1}\left(y^{\prime}\right)=\{u, v\}$ where $\kappa(u)=\kappa\left(y^{\prime}\right)=\kappa(v)$. Since $\left|\kappa\left(y^{\prime}\right): k^{\prime \prime}\right|=\frac{1}{2}\left|\kappa\left(y^{\prime}\right): k^{\prime}\right|$, the induction hypothesis shows that $u$ is good. Therefore $y^{\prime}$ is good by Lemma 8 and hence $y$ is good by Lemma 7 .

The corresponding result for the affine case now follows easily.

Corollary. Let $V \subset \mathrm{A}_{k}^{d+1}$ be the affine hypersurface defined by $q=1$ where $q$ is a quadratic form over a field $k$ of characteristic not 2 and $d>0$. If $q$ is nonisotropic and represents 1 then $A_{0}(V)=\mathbb{Z} / 2 \mathbb{Z}$. In all other cases $A_{0}(V)=0$.

Proof. Let $X \subset \mathbb{P}_{k}^{d+1}$ be defined by $q-y^{2}=0$ and let $X_{\infty}=X \cap(y=0)$. Then $V=X-X_{\infty}$ and we have an exact sequence $A_{0}\left(X_{\infty}\right) \rightarrow A_{0}(X) \rightarrow A_{0}(V) \rightarrow 0$. Since $\operatorname{deg}: A_{0}(X) \rightarrow \mathbb{Z}$ is injective by the theorem, it follows that $A_{0}(V)=$ $\operatorname{deg} A_{0}(X) / \operatorname{deg} A_{0}\left(X_{\infty}\right)$, which is zero unless $X$ has a rational point and $X_{\infty}$ does not, in which case it is $\mathbb{Z} / 2 \mathbb{Z}$.

\section{REFERENCES}

1. W. Fulton, Interesection theory, Ergeb. Math. Grenzgeb. (3), 2, Springer, Berlin-New York, 1984.

2. T. Y. Lam, Algebraic theory of quadratic forms, Benjamin Cummings, Reading, Mass. 1973.

3. R. G. Swan, Vector bundles, projective modules, and the K-theory of spheres, Proc. of the John Moore Conference, Algebraic Topology and Algebraic $K$-Theory (W. Browder, ed.) Ann. of Math. Stud. 113 (1987), 432-522.

4. __ K-theory of quadric hypersurfaces, Ann. of Math. (2) 122 (1985), 113-153.

Department of Mathematics, University of Chicago, Chicago, Illinois 60637 